\title{
Susceptibility characterization of beam pipe radiated noise for the PXD detector in Belle II experiment
}

\author{
M.Iglesias \\ Instituto Tecnológico de Aragón \\ ITAINNOVA \\ Zaragoza, Spain \\ miglesias@itainnova.es \\ F.J. Arcega \\ Departamento de Ingeniería Eléctrica \\ Universidad de Zaragoza \\ Zaragoza, Spain \\ A. Pradas \\ Instituto Tecnológico de Aragón \\ ITAINNOVA \\ Zaragoza, Spain
}

\author{
P.Leitl \\ Max Planck Institute for Physics \\ Werner-Heinsenberg-Institute \\ Munich, Germany
}

H-G. Moser

Max Planck Institute for Physics

Werner-Heinsenberg-Institute

Munich, Germany

I. Echeverría

Instituto Tecnológico de Aragón

ITAINNOVA

Zaragoza, Spain

F. Arteche

Instituto Tecnológico de Aragón

ITAINNOVA

Zaragoza, Spain

\author{
F.Müller \\ Max Planck Institute for Physics \\ Werner-Heinsenberg-Institute \\ Munich, Germany
}

\author{
C. Kiesling \\ Max Planck Institute for Physics \\ Werner-Heinsenberg-Institute \\ Munich, Germany \\ F.J. Piedrafita \\ Instituto Tecnológico de Aragón \\ ITAINNOVA \\ Zaragoza, Spain
}

\begin{abstract}
The new Pixel Vertex Detector (PXD) used in the upgrade of the high energy physics experiment Belle II is based on the DEPFET technology. Since the PXD is $2 \mathbf{~ m m}$ far from the beam pipe, the effects of radiated interferences may be taken into account. Though the EM wave associated to the beam is very well confined (skin depth), the beam pipe is grounded to the accelerator and it may have noise currents on its external face due to pumps, auxiliary electronics, power converters, etc. which may produce radiated noise (H field). This analysis is part of the EMC approach that covers the analysis of the emissions and immunity characteristics, as well as the coupling phenomena and grounding issues to define the susceptibility levels required to ensure the successful integration of the detector and, specifically, to achieve the designed performance of the front-end electronics.
\end{abstract}

Keywords -Electromagnetic compatibility; grounding; Susceptibility; particle tracking detectors; PXD

\section{INTRODUCTION}

The Belle II collaboration is aiming for new frontiers in the precision measurements of $\mathrm{B}$ meson decays with an upgraded detector system at the High Energy Accelerator Research Organization (KEK) in Tsukuba, Japan. The foreseen increase in luminosity of the SuperKEKB accelerator by a factor of 40 compared to the previous machine requires significant improvements in the detectors. To cope with the high density of charged particles close to the interaction point and still achieve a resolution of the decay vertices of the $\mathrm{B}$ mesons in the order of $10 \mu \mathrm{m}$ a new vertex detector based on two different technologies has been installed in Belle II experiment [1]. The vertex detector consists of several modules and front-end electronics (FEE) arranged cylindrically in six layers around the interaction point. The first two layers are based on DEPFET pixel technology [2] whereas the outer four layers are based on double-side silicon strip technology [3].
The vertex detector is arranged within the BELLE II experiment (Fig. 1), a hermetic detector system which allows the reconstruction of particle tracks from collisions, and performs the measurement of energy, momentum, particle type and charge of the produced particles.

The DEPFET detector involves a complex electrical system comprising the Front End Electronics (FEE), the Power Supplies, cables and connectors. The FEE are very sensitive to EM noise and they may radiate through the clock and other high frequency signals $[4,5]$.

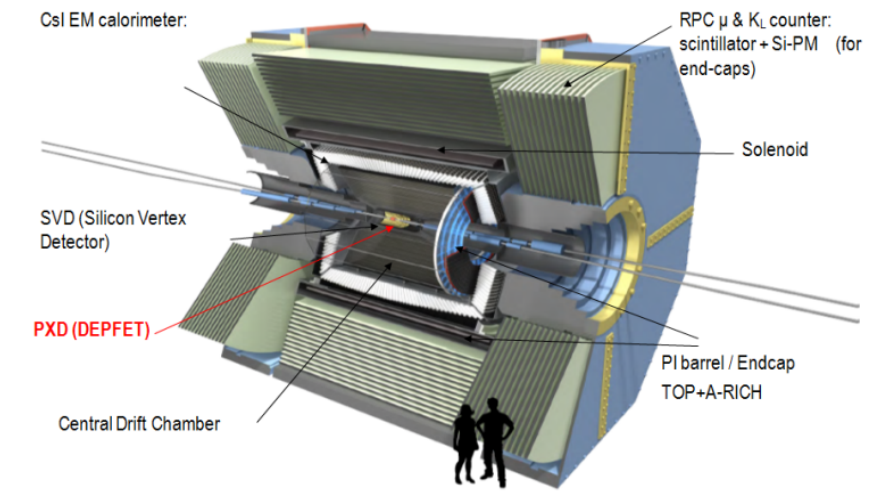

Fig. 1. Belle II experiment with PXD detector.

The Power Supplies can produce EM noise as well, and the cables and connectors may propagate EM noise inside and outside the FEE [6]. The grounding and shielding strategy for the DEPFET detector modules is part of an EMC strategy for the characterization of the EM environment of the PXD and ensures the correct integration of the FEE. As opposite to a PCB where the ground plane is usually clearly defined, in the case of the PXD the grounding is more complex. The scope of the grounding and shielding plans are to define the safety grounding, the signal or ground 
reference plane and the grounding topologies. These issues will be used to verify electrical safety issues and identify possible ground loops, Electromagnetic Interference (EMI) sources and EMI victims. In this context the beam pipe is considered as important noise source which might affect the system. The beam pipe is located only $2 \mathrm{~mm}$ far from the beam pipe and the EM field created by the beam is therefore considered as a significant source for induced noise.

Previous studies [7, 8] have shown than the distributions of the perturbations in Silicon detectors are governed by near field interactions. In the case under study, the interference coupling mechanism is dominantly inductive, and the perturbation than can arise will be as a consequence of the non-uniform magnetic fields generated by the common mode currents flowing through the external face of the beam pipe.

Fig. 2 depicts the PXD with the FEE and supporting cooling blocks mounted around the beam pipe.

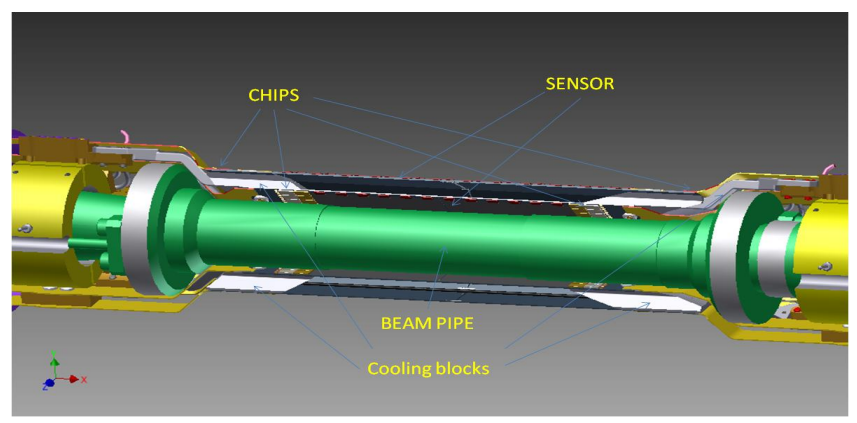

Fig. 2. Beam pipe and PXD detector.

This paper presents a characterization of the susceptibility level of the FEE to the coupled radiated disturbances from the beam pipe, identifying the key elements for the noise degradation, as well as the study of the coupling effects in different parts of the detector depending on the position of the noise source with several configurations of the detector shielding.

This is carried out through a set of measurements in which noise is injected into the system, and the noise degradation is quantified allowing the extraction of the susceptibility curves of the sub-detector. It is the first time that this kind of tests have been performed in a pixel detector based on DEPFET technology [2]. The outcome of this study will provide information to identify the most susceptible parts of the system to electromagnetic noise, which will help to integrate the PXD sub-detector in the Belle II experiment.

The work presented in this paper is performed within the project (AIDA2020) by mean collaboration between Max Plank Institute for Physics and ITAINNOVA. This project brings together the leading European research infrastructures in the field of detector development.

\section{SYSTEM DESCRIPTION}

The PXD modules combine an active area of DEPFET pixels, the readout and steering chips and a landing area for a flexible interconnect kapton cable. Each DEPFET pixel consists of a fully depleted silicon substrate and is equipped with a p-channel MOSFET structure and an internal gate where the electrons liberated by traversing charged particles are collected [9]. The readout electronics consists of three types of ASICs:
- DCD: Drain Current Digitizer, which digitizes the DEPFET currents from a row of pixels.

- DHP: Digital Handling Processor, which performs the digital processing like zero-suppression of empty pixels.

- Switcher: which activates and controls the pixel rows.

While the Switchers are located along the side of the DEPFET sensor on a $2 \mathrm{~mm}$ wide non-thinned rim, the DCDs and the DHPs are located at the end of the module outside of the acceptance region. The readout and control ASICs of the PXD sub-detector are bump-bonded on the rigid edges of the silicon substrate, whereas in the region of the active pixel matrix the substrate is thinned down to 75 $\mu \mathrm{m}$. Fig. 3 shows the view with the geometric arrangement of the PXD module.

The PXD module used in these tests consist of a sensor in which the pixels are arranged in a $768 \times 250$ pixel matrix. To drive these pixels, a group of 6 Switchers is used, each controlling 32 gates. As a result, a total of 192 gates are connected to the pixel matrix to active each transistor. On the other hand, currents are extracted into the 4 DCDs with a total of 1000 drains.

Due to this electronic connection scheme only one gate line is required to activate four geometrical rows of the DEPFET matrix so that a total of 1000 pixels can be read out at the same time. This four-fold parallel readout is called rollingshutter mode. After the digitization of the currents the internal gates are cleared again. The readout process of one gate is completed in about $104 \mathrm{~ns}$ summing up to a total readout time of $20 \mu$ s for the complete sensor matrix.

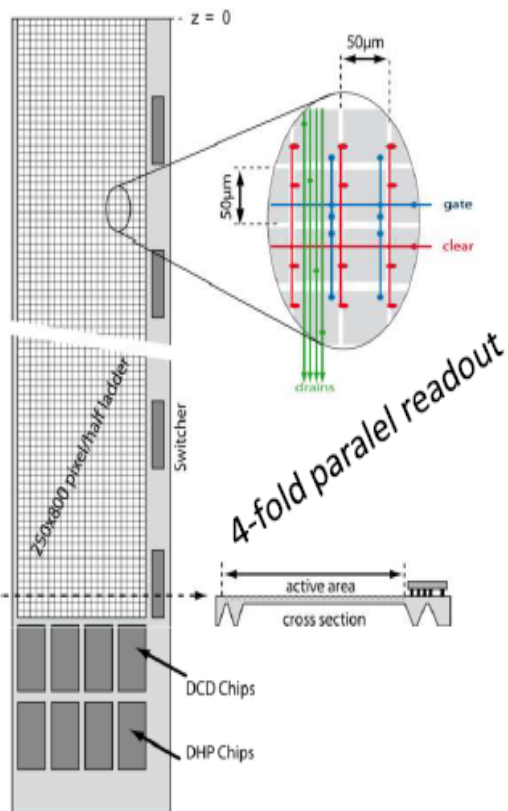

Fig. 3. DEPFET sensor (768x250 pixel matrix)

\section{EXPERIMENTAL SETUP}

The experimental setup is designed such that the FEE and the auxiliary equipment exhibit during the test a configuration as close as possible to the final one, as depicted in Fig. 4. 
The FEE and the auxiliary equipment are placed on a copper plane as suggested by IEC 61000-4-6 [10]. This copper plane is the reference ground plane. The Bulk Current Injection (BCI) method [11] consists of injecting a sine wave current through the cables as Common Mode (CM) perturbation $[12,13]$ and the FEE output signal is measured by its acquisition system.

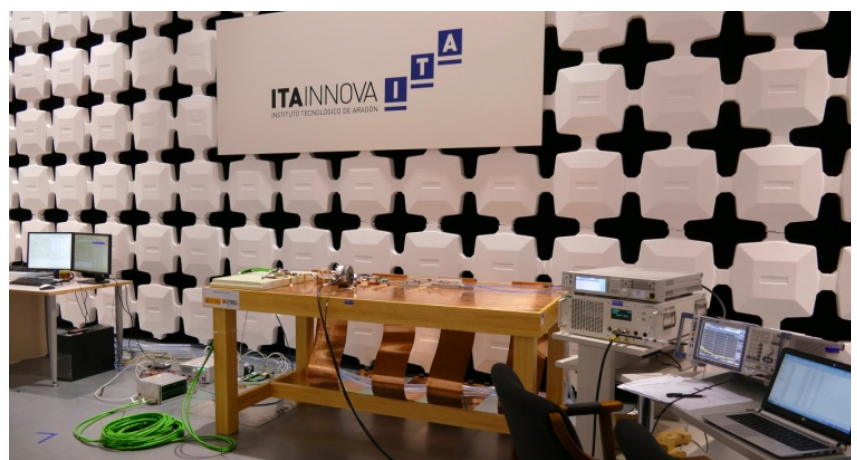

Fig. 4. General setup.

In this study, radiated noise like the one produced by the pipe is emulated by forcing a current to flow through a metallic structure located close to the detector. In this case, an aluminum braid is used as conducting path for the injected currents. The level of the injected signal is monitored using an inductive current clamp and a spectrum analyzer.

The sine wave injected at different frequencies $(100 \mathrm{kHz}$ to $400 \mathrm{MHz}$ ) and amplitudes will perturb the FEE by adding a noise component to the intrinsic thermal noise component of the PXD. The level of the signal injected is large enough to have a good signal-to-noise ratio at the input of the acquisition system without affecting the linearity of the overall FEE. For each of the selected frequencies, the current amplitude is set at a level high enough to produce a noticeable degradation in the noise mapping. Fig. 5 shows the setup implemented on the testing table.

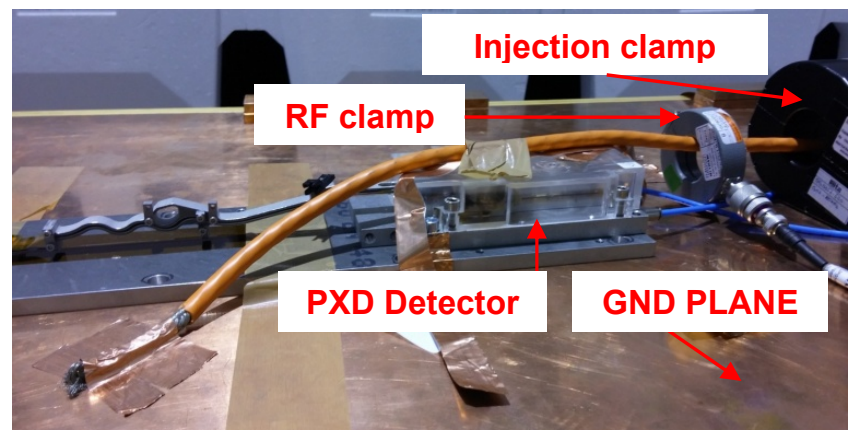

Fig. 5. Beam pipe noise test.

\section{NOISE DISTRIBUTION}

The noise that appears in the PXD sensor is evaluated via RMS value of each pixel computed over 300 measuring frames, which can be visualized on a 192 x 1000 color map matrix where the Y Axis represents the 192 gates controlled by six Switchers, and the X Axis represents the 1000 drains connected to the four DCDs. This representation allows dividing the noise map into four columns (each corresponding to a DCD) and six rows (each corresponding to a Switcher). Fig. 6 shows the map when no noise is injected. Analog to Digital units are used for the noise distribution representation in each pixel [14]

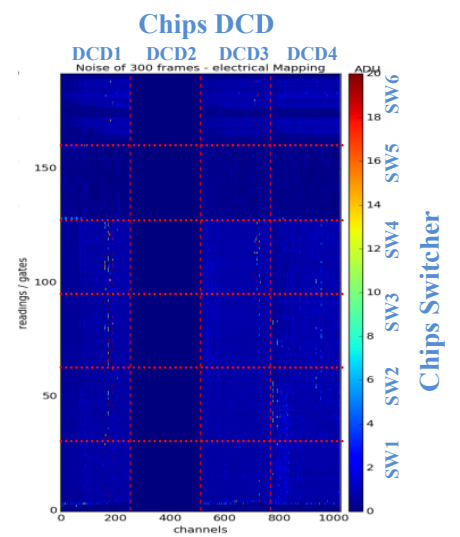

Fig. 6. Noise electrical map with no injection (thermal noise).

The injected currents to the FEE affect its performance and the perturbation depends on the amount of the interference current coupled to the sensitive areas of the FEE. Fig. 7 and Fig. 8 show the variation of the RMS values of the digitized voltage at the output of the FEE and with the same specific perturbing current level $(56 \mathrm{~mA})$ for each channel, but at 2 different frequencies (5 MHz and $15 \mathrm{MHz}$ respectively). The RMS output value includes both the intrinsic thermal noise of the FEE and the noise due to the injected current. The effect of the perturbation does not distribute equally for all the channels of the same sensor. The most susceptible pixels are located in the right edges of the chips. This aspect is common to all of them and shows than the pixels read by the external channels of each DCD chips are the noisiest. This distribution is not related only with each DCD but it also shows influence with the Switcher chips. In fact, these distributions are generated by the variation of the coupling circuit impedances at the input of each DCD chip, in a way that noise is coupled by the loop created through the Switcher chips located on the right side of the pixel active matrix.

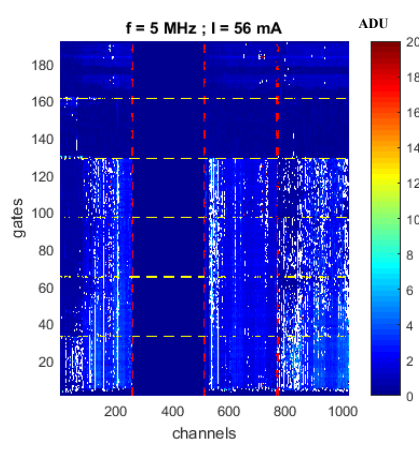

Fig. 7. Noise electrical map with injection $(5 \mathrm{MHz}$ and interference current $56 \mathrm{~mA}$ )

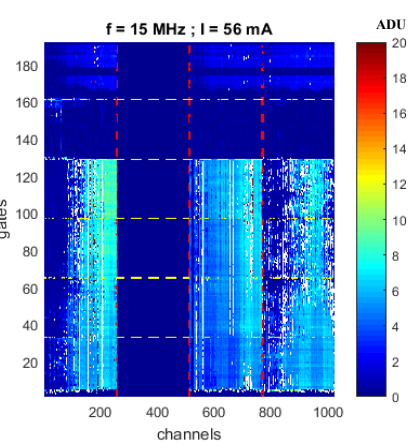

Fig. 8. Noise electrical map with injection $(15 \mathrm{MHz}$ and interference current $56 \mathrm{~mA})$
As we have observed, the noise coupled in the system is distributed in a different way, depending on the frequency and the level of injected current. The correct analysis of the 
system susceptibility requires the use of transfer functions, which are presented in the next section.

\section{NOISE TRANSFER FUCTION}

The susceptibility is quantified using transfer functions (TF). These functions relate the noise that is injected into the cable (current produced by the injection clamp) with the noise disturbance after the readout process (counts). The noise TF is defined as the relation between the noise RMS variation in the output voltage $\left(\mathrm{V}_{\text {out }}\right)$ and the Injected Common Mode current $\left(\mathrm{I}_{\mathrm{CM}}\right)$ as measured on the test line with a current clamp (1). Since the current is injected at different frequencies, each frequency will yield a transfer function value. As this function must represent the noise contribution of the injected current, thermal noise (noise level when no injection is performed) must be subtracted (2) [5]:

$$
\begin{gathered}
T F_{C M}(f)=\frac{V_{\text {out }}}{I_{C M}} \\
V_{\text {out }}^{2}=V_{\text {measured }}^{2}-V_{\text {th }}^{2}
\end{gathered}
$$

If the TF is computed for each pixel (192000) at different injection frequencies, the coupling map of the sensor is obtained. Representing the electrical map of the sensor pixel arrangement in terms of connection to the DCD and Switchers, a transfer function color map can be obtained for each frequency, which shows the areas of the pixel in which noise transfer is higher. DCD2 chip area is not plotted since DCD was not operative during the tests. If this map is computed for each frequency, the plots in Fig. 9 are obtained. This figure shows the TF maps obtained at each frequency for the common mode tests performed.

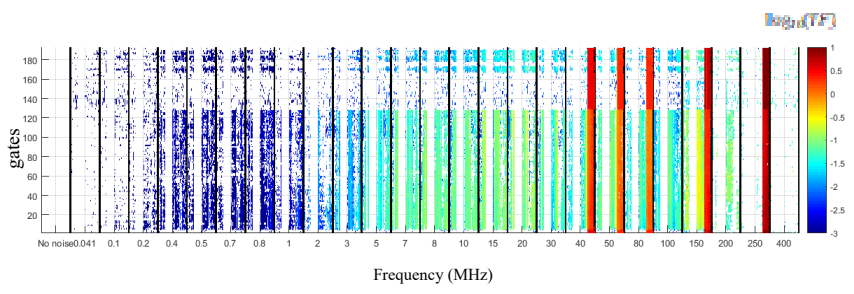

Fig. 9. TF maps for beam noise test.

If the sensor map is grouped into the area corresponding to the DCD1 and the transfer function is plotted along the frequency axis for each pixel, the graph in Fig. 10 is obtained. In this figure, the average function is computed among all the pixels (magenta trace), resulting in the average transfer function of the DCD1 area.

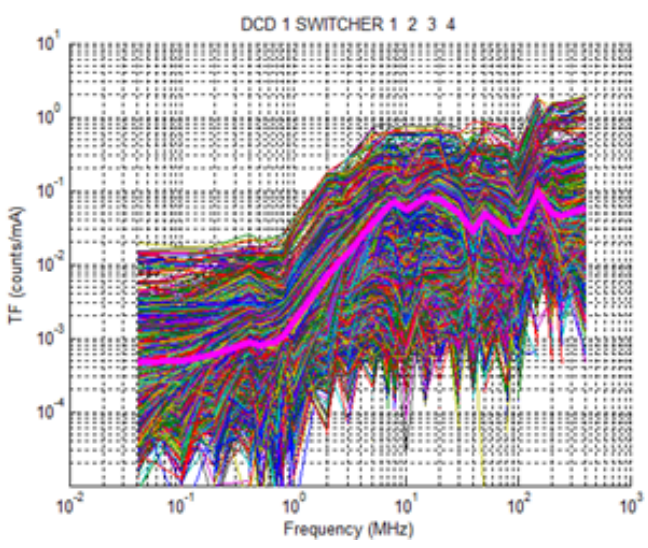

Fig. 10. TF per pixel in DCD1 sensor area

Once the average transfer function is computed for each DCD group, the plot in Fig. 11 is obtained. These average transfer functions can be fitted to a mathematical model that will represent the FEE sensitivity to $\mathrm{CM}$ currents flowing in the external face of the beam pipe and it could be used to establish the noise compatible level of the PXD detector.

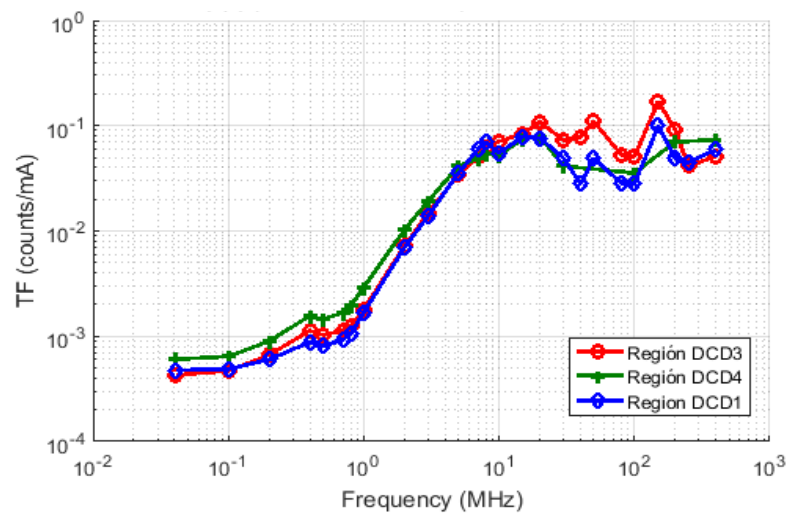

Fig. 11. Radiated noise average susceptibility TF per DCD chip sensor area

\section{LAYOUT EFFECTS}

Several modifications have been implemented on the shielding as well as the radiating cable position in order to study the coupling effects in different parts of the detector (kapton cable, pixel module). Fig. 12 shows the different test setups. First, the PXD module is covered with copper stripe to achieve a shielding effect (A), which later is improved by covering also the back side of the PXD box (B). Afterwards, one end of the radiating cable is moved apart from the Kapton cable (C), and then the shield underneath the cable is moved, leaving this part of the cable exposed (D). In (E) the copper enclosure is removed, leaving only the shield underneath the cable on top of the box. In (F), the cable is moved to be located on one side of the Kapton cable, parallel to it, and in $(\mathrm{G})$ the cable is located in front of the PXD box. Finally, the cable is positioned on top of the Kapton cable perpendicular to it $(\mathrm{H})$, and on top of the PXD sensor also perpendicular to it (I). This part of the study has been focused on a fixed frequency of $10 \mathrm{MHz}$ for the injection as previous results showed a higher susceptibility of the system at this frequency. For each configuration, the noise present in the system is measured in Analog Digital Units (ADU) [14, 15] and the results are displayed in table I. 


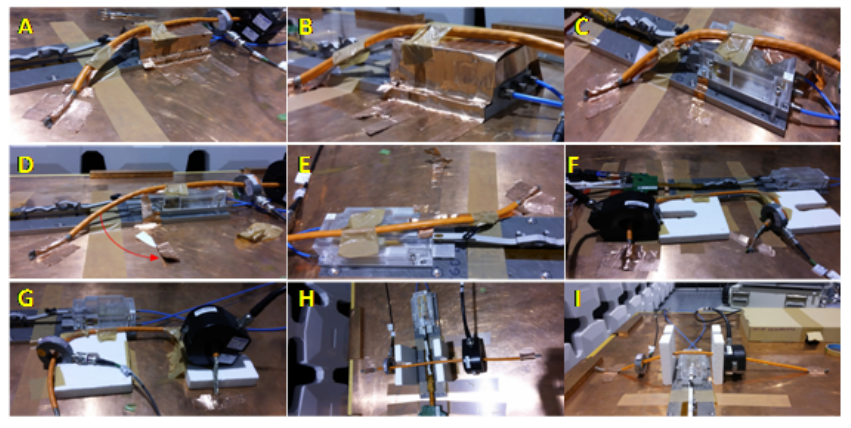

Fig. 12. Radiating cable configurations above the PXD module.

TABLE I. NOISE MEASURED IN PXD SENSOR

\begin{tabular}{|c|c|c|l|}
\hline $\begin{array}{c}\text { CONFI- } \\
\text { GURATION }\end{array}$ & $\begin{array}{c}\text { MEASURED } \\
\text { CURRENT } \\
(\mathrm{mA})\end{array}$ & $\begin{array}{c}\text { MEASURED } \\
\text { NOISE } \\
(\mathrm{ADU})\end{array}$ & $\begin{array}{l}\text { CABLE CONFIGURATION } \\
\text { ABOVE THE PXD MODULE }\end{array}$ \\
\hline $\mathbf{A}$ & $\mathbf{5 6}$ & 3 & With shielding \\
\hline $\mathbf{B}$ & $\mathbf{5 6}$ & 1 & Improving shielding \\
\hline $\mathbf{C}$ & $\mathbf{5 6}$ & 1.2 & $\begin{array}{l}\text { Shield only } \\
\text { underneath cable }\end{array}$ \\
\hline $\mathbf{D}$ & $\mathbf{5 6}$ & 3 & $\begin{array}{l}\text { Moving the shield so } \\
\text { that kapton area is } \\
\text { exposed }\end{array}$ \\
\hline $\mathbf{E}$ & $\mathbf{5 6}$ & 2 & $\begin{array}{l}\text { Removal of shield part } \\
\text { only on the sensor } \\
\text { cage }\end{array}$ \\
\hline $\mathbf{F}$ & $\mathbf{5 6}$ & 4 & $\begin{array}{l}\text { Cable parallel to } \\
\text { kapton }\end{array}$ \\
\hline $\mathbf{G}$ & $\mathbf{1 5 8}$ & 1.8 & $\begin{array}{l}\text { Cable in front of } \\
\text { sensor }\end{array}$ \\
\hline $\mathbf{H}$ & $\mathbf{1 5 8}$ & 1.2 & $\begin{array}{l}\text { Cable perpendicular } \\
\text { to kapton }\end{array}$ \\
\hline $\mathbf{I}$ & $\mathbf{1 5 8}$ & 1.2 & $\begin{array}{l}\text { Cable perpendicular } \\
\text { above the sensor }\end{array}$ \\
\hline
\end{tabular}

As the chart shows, higher levels of noise are detected when the radiating cable is located near the Kapton cable. Depending on the total exposed Kapton cable area to the interference magnetic field, higher level of coupled noise is obtained. Also when the shielding over the Kapton is removed, an increase in the noise is observed, while hardly any increase of noise is observed when the radiation is applied near the PXD box. The shield effectiveness comes as a result of induced currents flowing in a way that they oppose the magnetic field perturbation. Depending of the shielding area (covering the PXD module), the impedance of the path is decreased and the effect of the coupled noise is minimized.

This shows a noticeable higher susceptibility to radiating noise of the Kapton part of the sensor in comparison with the PXD module.

\section{CONCLUSIONS}

The susceptibility of a PXD sensor to beam pipe radiation has been studied. In order to emulate this noise, a special test set-up has been prepared. A noise current is forced to flow through a near cable in a way that it creates a magnetic field similar to the one radiated by noise current running through the external face of the beam pipe. The three tested DCD areas present a similar profile. As it can be seen, the noise coupling increases with the frequency from $100 \mathrm{kHz}$ until $10 \mathrm{MHz}$, where the function becomes flat. Several configurations have been tested in order to analyze this effect in detail, showing that the kapton area is more susceptible than the sensor area. As the chart shows, higher levels of noise are detected when the radiating cable is located near the Kapton cable. After removing the shielding above the kapton an increase in the noise is observed, while hardly any increase of noise is observed when the radiation is exposed near the PXD box. This shows a noticeable higher susceptibility to radiating noise of the Kapton part of the sensor in comparison with the PXD sensor itself.

\section{ACKNOWLEDGMENTS}

This Project has received funding from Ministerio de Economia (Spain) under grant agreement FPA2014-55295C3-3R (CMSRUN2) and FPA2017-85155-C4-3R (CMSRUN2B), from Advanced European Infrastructures for Detectors at Accelerators (AIDA2020) under grant agreement H2020-INFRAIA-654168 and from MoU between ITAINNOVA and Max Planck Institute for Physics.

\section{REFERENCES}

[1] P Križan, "Particle identification at Belle II", Journal of Instrumentation, Volume 9, July 2014

[2] F Lütticke, "The Belle II DEPFET pixel detector", Journal of Instrumentation, Volume 8, February 2013

[3] M.Friedl, “The Belle II Silicon Vertex Detector", Physics Procedia. Volume 37, 2012.

[4] F.Arteche, FJ Arcega, Cesteban, C Rivetta, M.Iglesias. "Detector noise suceptibility issues for the future generation of High Energy Physics Experiments". 13th Topical Workshop on Electronics for Particle Physics 2008. Proceedings International Topical Workshop on Electronics for Particle Physics. (ISBN:97-892-9083324-6), vol1, pp 533-538. September 2008

[5] F.Arteche, C.Rivetta. "EMC Phenomena in HEP Detectors: Prevention and Cost Savings" SNIC Sypmosium, Standford, California. 3-6 April 2006.

[6] F.Arteche, C.Esteban, M. Iglesias,C. Rivetta, F.J. Arcega, I. Vila. "DC/DC switching converter based power distribution vs Serial power distribution: EMC strategies for SLHC tracker up-grade." International Topical Workshop on Electronics for Particle Physics (ISBN: 978-92-9083-335-2), Vol 1, pp 384-389.

[7] Arteche, F, Esteban, C \& Rivetta,C "Interference coupling mechanisms in Silicon Strip Detectors-CMS tracker wings: A learned lesson for SLHC'. Topical Workshop on Electronics for Particle Physics, Paris, France, pp.394-399 (CERN-2009-006)

[8] F. Arteche, C. Rivetta, M.Iglesias, I.Echeverría. "Analysis and quantification of coupling mechanisms of external signal perturbations on silicon detectors for particle physics experiments" 2016 ESA Workshop on Aerospace EMC (ISBN:978-9-2922-1303-9)

[9] J. Kemmer and G. Lutz. "New detector concepts". Nucl. Instr. Meth. Phys. Res. A 253.3 (1987), p. 365. Issn:0168-9002

[10] IEC 61000-4-6:2013: Electromagnetic compatibility (EMC). Part 4-: Testing and measurement techniques. Immunity to conducted disturbances, induced by radio-frequency fields

[11] ISO 11452-4 (2005-04-01): Road Vehicles-Component test methods for electrical disturbances from narrowband radiated electromagnetic energy. Part 4: Bulk current injection (BCI)

[12] A.Kempski, R. Smolenski, "Decomposition of EMI noise into Common and Differential noise in PWM inverter drive system", Electrical Power Quality and Utilisation Journal, Vol. XII, No. 1, 2006

[13] L.Youn Hee, A. Nasiri, "Conductive CM and DM Noise Analysis of Power Electronic Converters in Electric and Hybrid Electric Vehicles", 2007 IEEE Vehicle Power and Propulsion Conference, pp. 392 - 398, Arlington (EEUU), 9-12 Sep. 2007 
[14] Z. Dolezal (editor) et al. "Belle II technical design report. Technical Report 2010” KEK, Nov. 2010. Url arXiv:1011.03”

[15] M.Friedl, "The Belle II Silicon Vertex Detector", Physics Procedia. Volume 37, 2012 